Gut and Liver, Vol. 10, No. 2, March 2016, pp. 166-176

\title{
The Role of Mesothelial Cells in Liver Development, Injury, and Regeneration
}

\author{
Ingrid Lua and Kinji Asahina
}

Southern California Research Center for ALPD and Cirrhosis, Department of Pathology, Keck School of Medicine, University of Southern California, Los Angeles, CA, USA

Mesothelial cells (MCs) cover the surface of visceral organs and the parietal walls of cavities, and they synthesize lubricating fluids to create a slippery surface that facilitates movement between organs without friction. Recent studies have indicated that MCs play active roles in liver development, fibrosis, and regeneration. During liver development, the mesoderm produces MCs that form a single epithelial layer of the mesothelium. MCs exhibit an intermediate phenotype between epithelial cells and mesenchymal cells. Lineage tracing studies have indicated that during liver development, MCs act as mesenchymal progenitor cells that produce hepatic stellate cells, fibroblasts around blood vessels, and smooth muscle cells. Upon liver injury, MCs migrate inward from the liver surface and produce hepatic stellate cells or myofibroblast depending on the etiology, suggesting that MCs are the source of myofibroblasts in capsular fibrosis. Similar to the activation of hepatic stellate cells, transforming growth factor $\beta$ induces the conversion of MCs into myofibroblasts. Further elucidation of the biological and molecular changes involved in $\mathrm{MC}$ activation and fibrogenesis will contribute to the development of novel approaches for the prevention and therapy of liver fibrosis. (Gut Liver 2016;10:166176)

Key Words: Glisson's capsule; Hepatic stellate cells; Liver fibrosis; Mesothelial-mesenchymal transition; Myofibroblasts

\section{INTRODUCTION}

Hepatocytes occupy approximately $80 \%$ of the total liver volume and play essential roles in metabolism. In mice, the liver consists of median, right, left, and caudate lobes. In each lobe, the liver parenchyma is covered by a serous membrane (serosa) that comprises a single layer of mesothelial cells (MCs). MCs are easily recognized in liver sections under the microscope, based on their location on the liver surface and their flat morphology, but the presence of MCs is often ignored. Accordingly, little is known about their roles in the liver. Recent studies have suggested that MCs are not only a simple barrier of the liver surface but that they also actively contribute to liver development and injury. In this article, we first introduce basic knowledge of MC biology, and then we review their characteristics and functions in liver development, fibrosis, and regeneration.

\section{GENERAL FEATURES OF THE MESOTHELIUM}

The name of MCs comes from their presence in the epithelial lining of mesodermic cavities. ${ }^{1,2}$ As this name implies, MCs are mesodermal in origin and lie on the organ surfaces as single epithelial sheets. MCs are separated from underlying fibroblasts by the basal lamina. The mesothelium covers the surface of the visceral organs, including the heart, respiratory tract (lung), digestive tract (liver, stomach, and intestine), omentum, and ovaries. ${ }^{3}$ It also covers the parietal wall of the pleura, pericardium, peritoneum, and tunica vaginalis. MCs are flat epithelial cells that form epithelial sheets with tight junctions, adherence junctions, gap junctions, and desmosomes. ${ }^{4}$ MCs exhibit a squamous epithelial cell shape in normal organs, and they express both epithelial cell and mesenchymal cell markers. MCs secrete a lubricating fluid to create a slippery surface that facilitates movement between organs without friction. MCs synthesize decorin and biglycan, and they are a major source of proteoglycans in the peritoneal fluid. ${ }^{5}$ MCs express cell adhesion molecules, such as intercellular adhesion molecule 1 (ICAM-1) and vascular cell adhesion protein 1 (VCAM-1), and cytokines, such as SDF-1/ CXCL12, MCP-1/CCL2, and IL-8, and they facilitate inflammatory reactions via the recruitment of monocytes. ${ }^{6,7}$ The mesothelium acts as a semipermeable barrier for water and solutes, and

Correspondence to: Kinji Asahina

Southern California Research Center for ALPD and Cirrhosis, Department of Pathology, Keck School of Medicine, University of Southern California, 1333 San Pablo St., MMR 402, Los Angeles, CA 90089-9141, USA

Tel: +1-323-442-2213, Fax: +1-323-442-3126, E-mail: asahina@usc.edu

Received on May 20, 2015. Revised on August 1, 2015. Accepted on August 18, 2015.

pISSN 1976-2283 eISSN 2005-1212 http://dx.doi.org/10.5009/gnl15226

(.) This is an Open Access article distributed under the terms of the Creative Commons Attribution Non-Commercial License (http://creativecommons.org/licenses/by-nc/4.0) which permits unrestricted non-commercial use, distribution, and reproduction in any medium, provided the original work is properly cited. 

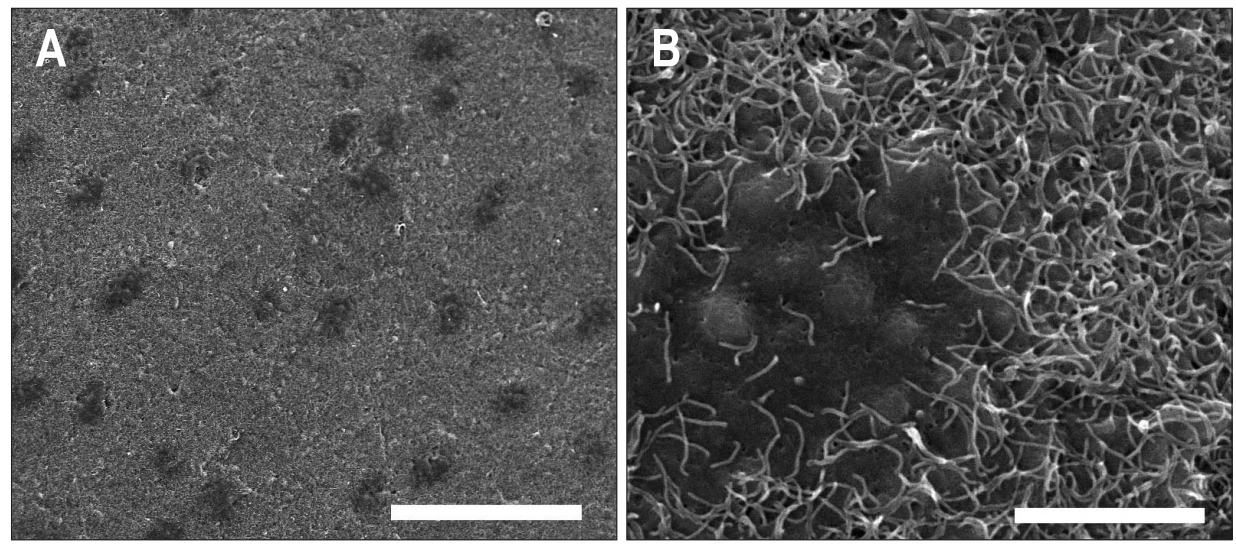

Fig. 1. Ultrastructure of the liver surface analyzed by scanning electron microscopy. Mesothelial cells on the surface of the normal adult mouse liver have microvilli protruding into the peritoneal cavity. The black regions represent areas having few microvilli above the nuclei. Scale bars, $50 \mu \mathrm{m}$ in (A) and $5 \mu \mathrm{m}$ in (B).
CD200

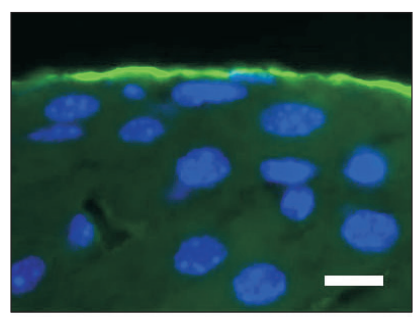

MSLN

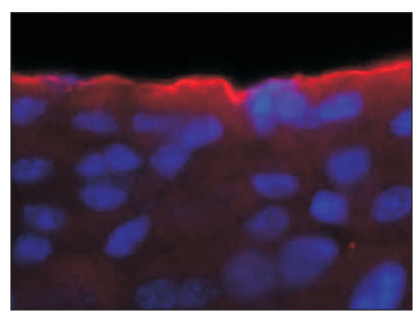

Cytokeratin

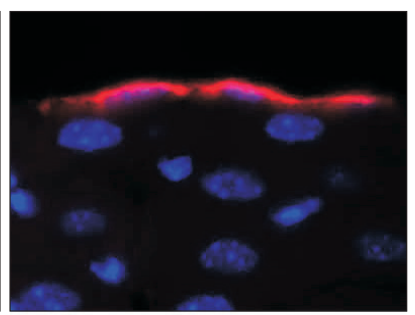

PDPN

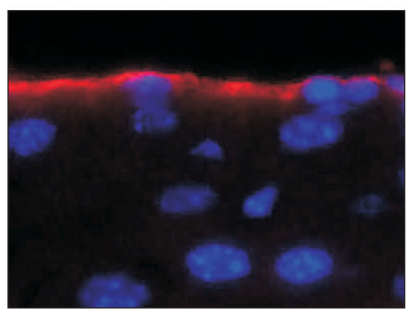

GPM6A

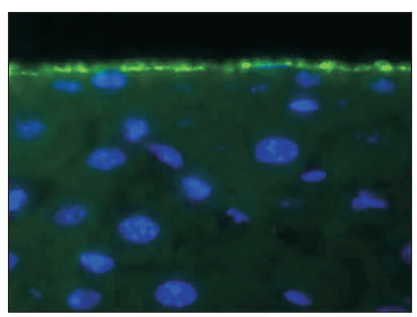

VIM

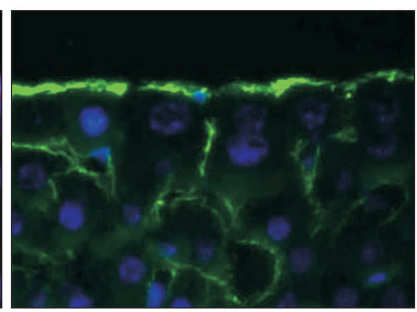

Fig. 2. Expression of markers in liver mesothelial cells (MCs). Immunohistochemistry shows the expression of CD200, cytokeratin, glycoprotein M6A (GPM6A), mesothelin (MSLN), podoplanin (PDPN), and vimentin (VIM) in MCs covering adult mouse livers. Scale bar, $10 \mu \mathrm{m}$. this ability has been exploited for peritoneal dialysis in patients with kidney failure. ${ }^{8}$

\section{CHARACTERISTICS OF LIVER MCS}

MCs form a single mesothelial cell layer that lines the liver surface and exhibit squamous epithelial cell morphology. ${ }^{9,10}$ MCs in the adult mouse liver have microvilli protruding into the peritoneal cavity (Fig. 1). Microvilli are scarce from the membrane immediately above the nuclei of the MCs, although the reason for this scarcity is unclear. ${ }^{9,11}$ Liver MCs express ICAM-1 and VCAM-1 on the microvilli, mediating the binding of monocytes onto MCs due to stimulation by lipopolysaccharide. ${ }^{11}$ MCs express cytokeratin 8 and 19, tight junction proteins and gap junction proteins. ${ }^{10,12,13}$ In the mouse liver, the expression of Ecadherin is not evident in MCs. ${ }^{10}$ Liver MCs express mesenchymal cell markers, such as vimentin (VIM) (Fig. 2). In addition to these markers, MCs express CD200, glycoprotein M6A (GPM6A), mesothelin (MSLN), and podoplanin (PDPN) (Fig. 2). ${ }^{10}$

In the mouse liver, the parenchyma is covered by a single layer of MCs and underlying capsular fibroblasts, also called

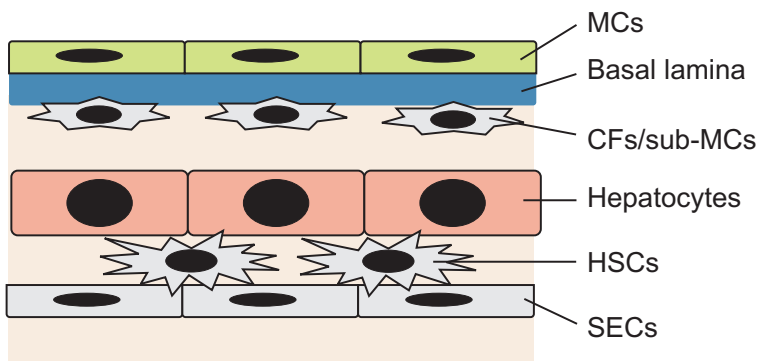

Fig. 3. Structure of the mesothelium in mouse liver tissue. Mesothelial cells (MCs) line up on the liver surface and form a single epithelial cell layer. The basal lamina separates the MCs from the underlying capsular fibroblasts (CFs)/sub-mesothelial cells (sub-MCs). Mouse livers show a single stratum of CFs beneath the MCs. Hepatic stellate cells (HSCs) reside in the space of Disse between hepatocytes and sinusoidal endothelial cells (SECs).

sub-mesothelial cells (sub-MCs) (Fig. 3)., ${ }^{911}$ Capsular fibroblasts synthesize collagen fibers and do not express MC markers. ${ }^{10,15}$ Hepatic stellate cells (HSCs) reside in the space of Disse between the hepatocytes and the sinusoidal endothelial cells, and HSCs store vitamin A lipids as retinyl esters (Fig. 3). ${ }^{16,17}$ Differing from 
HSCs, capsular fibroblasts beneath the MCs do not store vitamin A lipids in their cytoplasm. ${ }^{14}$ However, little is known about their characteristics and functions in the liver due to a lack of markers.

\section{DEVELOPMENT}

The development of the mesothelium has been studied mainly in chick embryos. During gastrulation, the mesoderm is formed between the ectoderm and endoderm. The mesoderm becomes the paraxial mesoderm, intermediate mesoderm, and lateral plate mesoderm. ${ }^{18}$ The paraxial mesoderm forms the somite and gives rise to skeletal muscle, cartilage, and the connective tissue of the skin. The intermediate mesoderm gives rise to the urogenital organs, including the kidneys. The lateral plate mesoderm gives rise to blood vessels and mesenchymal cells in the heart, digestive tract, body wall, and limbs. The mesothelium is derived from the lateral plate mesoderm. ${ }^{18}$ As the coelomic cavity develops in the lateral plate mesoderm, the mesoderm forms the splanchnic mesoderm and somatic mesoderm. The splanchnic mesoderm develops with the endoderm, and together they form the gut tube. During the gut organogenesis, mesodermal cells, exposing the coelomic cavity, become visceral MCs that cover the surface of the internal organs, including the lungs, liver, and intestine. ${ }^{18,19}$ The somatic mesoderm underlying the ectoderm forms the wall of the cavity and gives rise to parietal MCs, which cover the surfaces of the body cavities. Thus, visceral and parietal MCs are separated during the formation of the coelomic cavity in the early developmental stage. In mouse embryogenesis, the nascent mesoderm, expressing mesoderm posterior 1 (MESP1), which is a basic helix-loop-helix transcription factor, contributes to visceral MCs in the liver. ${ }^{20,21}$ In contrast, MESP1+ mesoderm does not contribute to parietal MCs on the body wall of the peritoneal cavity, indicating distinct origins of the visceral and parietal MCs in mice. ${ }^{15}$ In chick embryos, MCs in the gut derive from resident mesenchymal progenitor cells. ${ }^{22}$ MCs seem to have organ-specific functions in each organ.

\section{DEVELOPMENT OF THE SEPTUM TRANSVERSUM MESENCHYME}

Liver epithelial cells originate from the definitive endoderm developed in the foregut (Fig. 4). A transplantation study in chick embryos showed that the foregut endoderm differentiates into liver in the presence of the cardiac mesoderm. ${ }^{23}$ In mouse embryos, the cardiac mesoderm secretes fibroblast growth factors and induces hepatoblasts from the foregut endoderm. ${ }^{24} \mathrm{In}$ addition, bone morphogenetic proteins from the septum transversum mesenchyme (STM) are required for the transformation of the endoderm into hepatoblasts. ${ }^{25}$ Hepatoblasts are bipotent liver progenitor cells, and they give rise to both hepatocytes and cholangiocytes. ${ }^{26,27}$ During liver development, hepatoblasts invade the STM and form a hepatic cord. ${ }^{28}$

The STM surrounding the foregut endoderm is heterogeneous, containing at least mesenchymal cells and endothelial cells. ${ }^{29}$ Although the STM is required for liver development, this transient mesenchymal cell population also contributes to heart development and diaphragm formation. Mesenchymal cells budding from the surface of the STM into the pericardial cavity are called proepicardial cells. ${ }^{30,31}$ They traverse the pericardial cavity, attach to the heart surface, and form a single layer of MCs on the heart surface, called the epicardium. Epicardial cells undergo epithelial-mesenchymal transition (EMT), migrate inward from the heart surface, and give rise to mesenchymal cells of the coronary vessels in chick embryos. ${ }^{32,33}$ In mouse embryos, proepicardial cells express T-box18 (TBX18) and Wilms tumor 1 homolog (WT1). Cell lineage tracing of TBX18+ or WT1+ proepicardial cells revealed their contributions to fibroblasts and
A

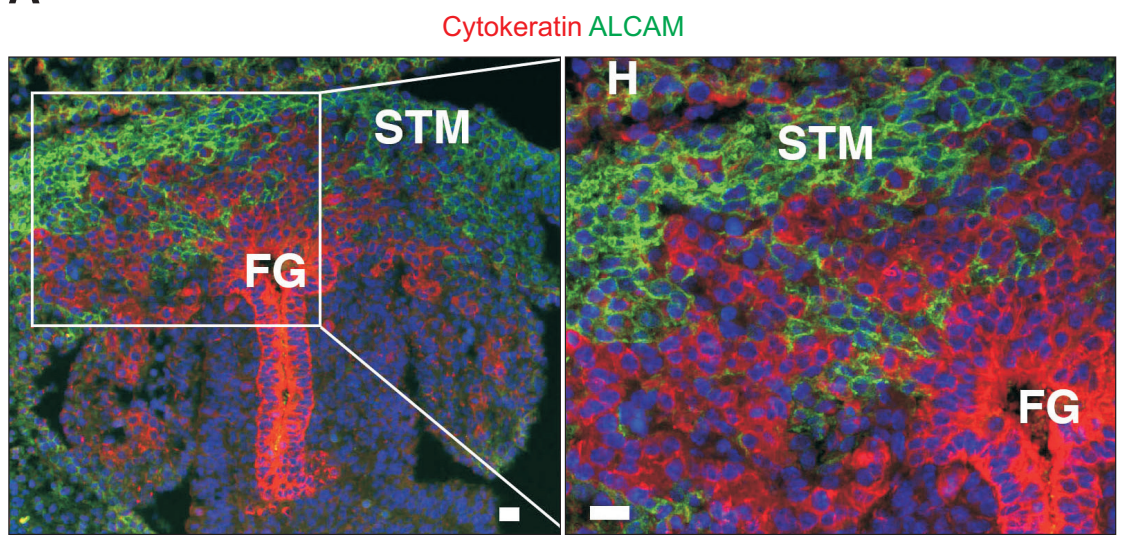

B

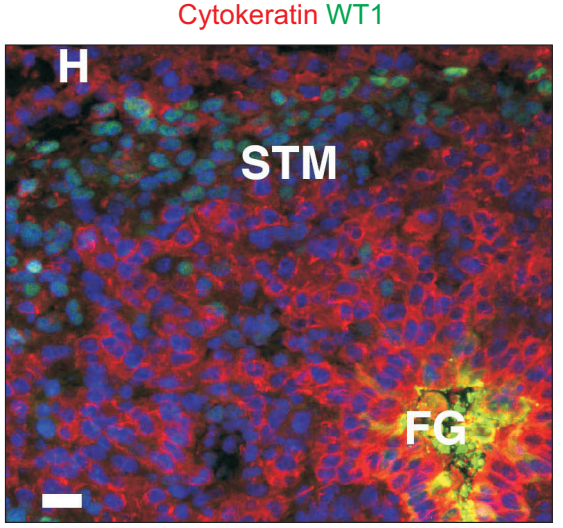

Fig. 4. Mouse liver development at embryonic day 10. Foregut endoderm (FG) differentiates into hepatoblasts that are positive for immunohistochemical staining of cytokeratin (red). Cytokeratin+ hepatoblasts invade the surrounding septum transversum mesenchyme (STM) and form liver buds. The STM expresses mesothelial cell markers, such as activated leukocyte cell adhesion molecule (A, green) and Wilms tumor 1 homolog (WT1) (B). WT1+ mesenchymal cells also differentiate into epicardial cells of the developing heart (H). Scale bar, $20 \mu \mathrm{m}$. 
smooth muscle cells in the developing heart. ${ }^{34,35}$ In addition, lineage tracing of Semaphorin3D (SEMA3D)+ and Scleraxis (SCX)+ proepicardial cells showed their contributions to coronary vascular endothelial cells, ${ }^{36}$ suggesting heterogeneity of the STM.

\section{CONTRIBUTION OF THE STM TO LIVER MESENCHYME}

Although the STM is known to be important for heart and diaphragm development, little is known about its cell lineage and function in liver development. A morphological study showed that hepatoblasts invade the surrounding STM and form a hepatic cord in mouse embryos (Fig. 4). ${ }^{28}$ During this process, mesenchymal cells seem to be trapped between growing hepatoblasts and become HSCs. Conditional cell-lineage tracing in mice revealed that WT1+ cells in the STM give rise to MCs on the liver surface and HSCs in the liver during liver development. ${ }^{29}$ GATA binding protein 4 (GATA4) is broadly expressed in both STM and endoderm at the onset of liver development. ${ }^{37}$ Rojas et al..$^{38}$ identified an enhancer of GATA4 which is specifically active in the lateral plate mesoderm and STM around E7.75-9.5 embryos but not in HSCs in the developing liver from E11.5. A lineage tracing study using the STM-specific GATA4 enhancer-Cre mouse showed that the STM contributes to 64\% of HSCs during liver development. ${ }^{39}$ The results of these studies indicate that the STM is the origin of HSCs during mouse liver development.

The STM expresses forkhead box F1 (FOXF1), GATA4, LIM homeobox protein 2 (LHX2), heart and neural crest derivatives expressed transcript 1 (HAND1), H2.0-like homeobox (HLX), mab-21-like 2 (MAB21L2), TBX18, zinc finger protein multitype 2 (ZFPM2/FOG2), and WT1..$^{29,37,40-46}$ Because the STM is the source of epicardium in the heart, of the diaphragm, and of MCs in the liver, deletion of the MAB21L2, TBX18, and ZFPM2 gene resulted in abnormal development of the heart, diaphragm, and liver. ${ }^{44-46}$ GATA4-null mouse embryos showed heart defects due to underdevelopment of the STM. ${ }^{37}$ Conditional deletion of GATA4 in the STM caused reduced liver size, precocious activation of HSCs, and abnormal deposition of ECM in embryonic livers, ${ }^{39}$ indicating that GATA4 regulates the quiescent phenotype of embryonic HSCs. Loss of deletion of $\beta$-catenin in MCs and HSCs expressing TWIST2 also caused abnormal activation of HSCs in embryonic livers. ${ }^{47}$ WT1 gene deletion resulted in abnormal development of the mesothelium, including of the liver, and impairment of hepatocyte proliferation. ${ }^{48-50}$ WT1 could regulate the differentiation of MCs into mesenchymal cells via retinoic acid signaling in developing livers. ${ }^{49,51}$ HLX-null embryos impaired hematopoiesis and resulted in liver hypoplasia. ${ }^{52}$ MCs in the embryonic liver express pleiotrophin (PTN) and midkine (MDK). ${ }^{48,53}$ PTN and MDK are secreted heparin-binding proteins, and they induce the proliferation of hepatoblasts in liver development. ${ }^{48}$

\section{MCs ACT AS MESENCHYMAL PROGENITOR CELLS DURING LIVER DEVELOPMENT}

Although the STM emerges as an aggregate of mesenchymal cells surrounding the foregut endoderm, it also expresses MC markers, such as activated leukocyte cell adhesion molecule (ALCAM/CD166) and WT1, on embryonic days (E) 9-10 in mouse embryos (Fig. 4). ${ }^{29}$ As the liver bud invades the STM, the surrounding mesenchyme becomes thinner, and flat MCs appear on the liver surface around E11.5. From E12.5, the MCs start to express cytokeratin, CD200, GPM6A, and PDPN, similar to MCs in adult livers. ${ }^{29} \mathrm{MCs}$ also express podocalyxin-like (PODXL) and MSLN. ${ }^{48}$ The expression of ALCAM and WT1 was observed, not only in MCs but also in sub-MCs beneath the MCs in the embryonic liver around E11-14. ${ }^{20}$ MCs and sub-MCs are separated by the basal lamina, which is composed of type IV collagen. Different from MCs, sub-MCs cells also express HSC markers, such as nerve growth factor receptor (NGFR/P75NTR) and platelet-derived growth factor receptor $\alpha$ (PDGFRA). HSCs and fibroblasts around the blood vessels express NGFR and PDGFRA but not MC markers, including ALCAM, PDPN, and WT1. SubMCs seem to be intermediate cells between MCs and HSCs in embryonic livers. ${ }^{54}$

A morphological study showed possible migration of MCs from the liver surface and differentiation into HSCs in mouse liver development. ${ }^{49}$ Conditional lineage tracing of WT1+ MCs/sub-MCs showed that they migrate inward from the liver surface and give rise to HSCs, portal fibroblasts, and smooth
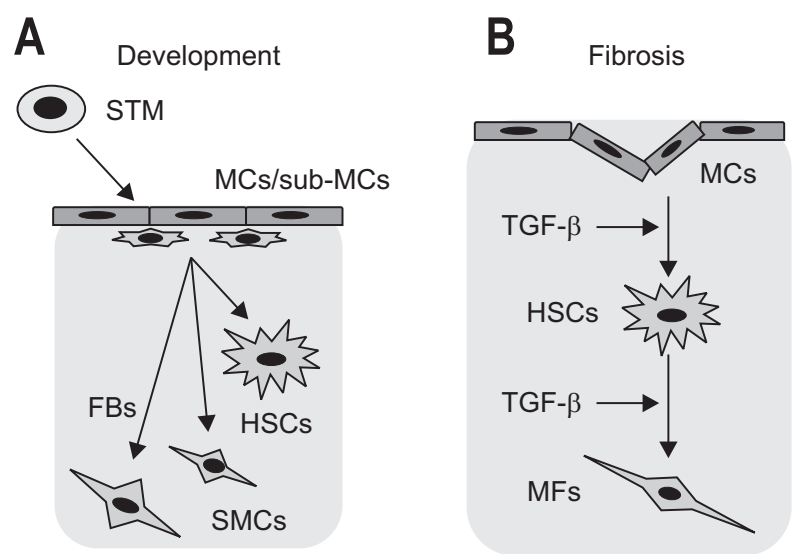

Fig. 5. Differentiation of mesothelial cells (MCs) in liver development and fibrosis. (A) During embryogenesis, mesoderm posterior 1 (MESP1)+ mesoderm gives rise to septum transversum mesenchyme (STM) and MCs. The MCs and underlying sub-MCs express Wilms tumor 1 (WT1). Cell-lineage tracing of WT1+ cells demonstrates their migration from the liver surface and differentiation into hepatic stellate cells (HSCs), fibroblasts (FBs) and smooth muscle cells (SMCs) around the blood vessels in mouse liver development. (B) Upon liver injury caused by $\mathrm{CCl}_{4}$ injection or bile duct ligation, MCs differentiate into myofibroblasts (MFs) or HSCs in adult mouse livers. Transforming growth factor $\beta$ (TGF- $\beta$ ) provokes the mesothelial-mesenchymal transition. 
muscle cells during mouse liver development (Fig. 5A). ${ }^{29}$ Lineage tracing of MSLN+ MCs also showed contributions of MCs to liver mesenchymal cells in embryos. ${ }^{55}$ These studies failed to detect the multidifferentiation of MCs into hepatoblasts, sinusoidal endothelial cells, and Kupffer cells. Thus, an HSC lineage from WT1+ MCs is distinct from that of other liver cell types in liver development. Possible migration of WT1+ sub-MCs was also documented in human liver development. ${ }^{56}$ The lines of evidence have indicated that MCs act as progenitor cells for liver mesenchymal cells during liver development.

Differentiation of MCs into HSCs has also been reported in chick and zebrafish livers. In chick embryos, after labeling MCs using a vital dye, these cells were found in the liver as HSCs or endothelial cells. ${ }^{57}$ In zebrafish, heart and neural crest derivatives expressed transcript 2 (HAND2)+ mesenchymal cells on the liver surface, presumably MCs, give rise to HSCs in liver development. ${ }^{58}$ These studies indicated that the migration and differentiation of MCs into HSCs are common processes during liver development in different species.

In addition to the developing liver and heart, differentiation of MCs into mesenchymal cells has been reported in the developing heart, lungs, and intestine. ${ }^{55,59}$ During lung development in mice, MCs migrate inward and give rise to smooth muscle cells and mesenchymal cells. ${ }^{60-62}$ Similar contributions have been reported in developing gut. ${ }^{22,63,64}$ In addition, WT1+ MCs were shown to differentiate into visceral fat in mice. ${ }^{65}$ These studies suggested that MCs are the source of mesenchymal cells in organogenesis.

\section{MESODERMAL ORIGIN OF MCs AND HSCs}

In adult livers, HSCs reside in the space of Disse and store vitamin A lipids as retinyl esters. ${ }^{16}$ Upon liver injury, HSCs are activated, and they differentiate into myofibroblasts expressing $\alpha$-smooth muscle actin (ACTA2). ${ }^{17}$ In addition to HSCs, there are portal fibroblasts around the bile duct, and they have been suggested to be another source of myofibroblasts in biliary fibrosis. ${ }^{66-68}$ MESP1 is a basic helix-loop-helix transcription factor expressed in the nascent mesoderm during gastrulation. ${ }^{69}$ MESP1+ mesoderm contributes to the primary and secondary heart fields and to other mesodermal cells during embryogenesis. Cell lineage tracing in MESP1-Cre and ROSA26 reporter mice showed that MESP1+ mesoderm gives rise to the STM before liver development. ${ }^{29}$ During liver development, MESP1+ cells contribute to MCs, HSCs, and portal fibroblasts around the vein. ${ }^{29}$ These cells differentiated into ACTA2+ myofibroblasts in fibrosis induced by $\mathrm{CCl}_{4}$ or bile duct ligation. ${ }^{21}$

\section{LIVER FIBROSIS AND MYOFIBROBLASTS}

Chronic liver injury caused by alcohol intake, drugs, hepatitis virus infection, and obesity results in fibrosis, and finally cir- rhosis. ${ }^{70-72}$ Upon liver injury, quiescent HSCs are activated, synthesize proinflammatory cytokines and extracellular matrices, and facilitate the regeneration of hepatocytes in injured livers. However, prolonged damage to the liver results in massive accumulation of collagen in the liver, leading to fibrosis and cirrhosis. HSCs have a fibroblastic morphology, and they express mesenchymal cell markers, such as desmin, Vim, and type I collagen. In addition, they express neural cell markers, such as glial fibrillary acidic protein, nestin, and NGFR. Although the neural crest was believed to be the origin of HSCs, based on their expression of neural markers, a cell lineage tracing study refuted this possibility. ${ }^{73}$ In addition, the presence of common progenitor cells for HSCs and hepatocytes in the embryonic liver has been proposed. ${ }^{747}$ However, as described above, the majority of HSCs have been shown to originate in mice from MESP1+ mesoderm..$^{20,21}$

Myofibroblasts seem to be heterogeneous, and they might derive from different sources during liver fibrosis. ${ }^{76-79}$ Although bone marrow cells have been shown to differentiate into HSCs or myofibroblasts, their contribution seems to be negligible in liver fibrosis. ${ }^{80-83}$ EMT of hepatocytes or cholangiocytes into myofibroblasts was proposed in liver fibrosis, but cell-lineage tracing studies have refuted this possibility in mice. ${ }^{84-92}$

In severely injured livers, oval cells, which are facultative stem cells, emerge in the portal area. Although oval cells are believed to be important for liver regeneration, their origin and roles have been controversial. ${ }^{93-95}$ HSCs were suggested to be the origin of oval cells in mice. ${ }^{96-98}$ However, lineage tracing of HSCs using lecithin-retinol acyltransferase (LRAT)-Cre mice did not find evidence of such conversion in different mouse injury models. ${ }^{99}$ Similarly, HSCs, portal fibroblasts, and MCs derived from MESP1+ mesenchymal cells did not contribute to hepatocytes, cholangiocytes, and oval cells, respectively, in mouse liver injury. ${ }^{21}$ These lineage tracing studies refuted the possibility of the conversion of HSCs into epithelial cells in liver injury.

\section{CONVERSION OF MCS INTO MYOFIBROBLASTS IN LIVER FIBROSIS}

In liver fibrosis, collagen deposition is observed around the blood vessels and along the sinusoid, depending on the etiology. Conditional lineage tracing of WT1+ MCs revealed that MCs migrated from the liver surface and gave rise to ACTA2+ myofibroblasts in $\mathrm{CCl}_{4}$-induced liver fibrosis. ${ }^{10} \mathrm{MC}$-derived myofibroblasts were detectable at up to $150 \mu \mathrm{m}$ in depth from the liver surface (Fig. 5B). Similar migration and differentiation of MCs were also observed in mouse livers after bile duct ligation (Fig. $5 \mathrm{~B}){ }^{10}$ Different from the $\mathrm{CCl}_{4}$ model, biliary fibrosis induces differentiation of MCs into HSCs, suggesting that MCs differentiate into myofibroblasts or HSCs in injured livers, depending on etiology. 


\section{CONTRIBUTION OF MCS TO FIBROSIS IN THE VISCERAL AND PARIETAL ORGANS}

Fibrosis is a scarring process in organs. Similar to the liver, fibrosis develops in different organs and myofibroblasts play pivotal roles in collagen deposition. ${ }^{100}$ Recent studies show the similar contribution of MCs to myofibroblasts in the peritoneum and lung. Patients who undergo peritoneal dialysis for kidney failure often develop peritoneal fibrosis. ${ }^{101-103}$ Prolonged exposure of a dialysis solution to the peritoneal cavity causes injury to MCs and induces conversion into myofibroblasts. Effluent of the dialysis solution from the peritoneal cavity contained MCs, showing a fibroblastic phenotype. ${ }^{104}$ Peritoneal fibrosis has been reproduced in mice by injection of dialysis solution or chemicals. Different insults to the peritoneal cavity cause injury to MCs on the body wall, as well as on the liver. In an experimental mouse model of peritoneal fibrosis, WT1+ MCs gave rise to myofibroblasts in both the body wall and liver. ${ }^{15}$ The contribution of MCs to myofibroblasts was approximately 16\%, and fibroblasts beneath the MCs might be another source. ${ }^{105}$ In the lungs, MCs also differentiate into myofibroblasts in idiopathic pulmonary fibrosis. ${ }^{106}$ These studies suggest that MCs are among the sources of myofibroblasts in the fibrogenesis of different organs.

MCs exhibit different phenotypes in different organs. Visceral MCs express autotaxin, and they have greater migration capacity than parietal MCs. ${ }^{107}$ Upon injury, peritoneal MCs on the body wall differentiate into myofibroblasts, and the MC layer disappears in mice. In contrast, liver MCs give rise to myofibroblasts that migrate beneath the MC layer, whereas the MC layer remains on the surface. It is unclear why visceral and parietal MCs behave differently upon injury. Recent studies have shown that visceral and parietal MCs are inherently different. In embryos, lung MCs are derived from the lung bud but not from other organs. ${ }^{22}$ Cell lineage tracing of MESP $1+$ mesoderm showed its contribution to MCs in the liver but not in the body wall. ${ }^{15}$

\section{MECHANISMS OF CONVERSION OF MCs INTO MESENCHYMAL CELLS}

MCs are isolated from the effluent of the peritoneal cavity or by the digestion of organs. In culture, primary MCs grow and form epithelial colonies of MCs. ${ }^{108,109}$ Recent studies have identified specific surface markers for liver MCs, such as GPM6A or PODXL, and these markers have allowed for the purification of MCs by fluorescence-activated cell sorting (FACS) or magneticactivated cell sorting (MACS). ${ }^{10,48}$ Primary liver MCs grew and formed epithelial colonies (Fig. 6A). However, MCs lost their epithelial phenotype over several passages and became fibroblastic cells spontaneously. ${ }^{10}$

Transforming growth factor $\beta$ (TGF- $\beta$ ) is a pleiotrophic factor known to be a strong inducer of EMT. ${ }^{110}$ In culture, TGF- $\beta$ treatment induced morphological changes of MCs into myofibroblasts (Fig. 6B). ${ }^{10}$ According to these changes, MCs increased the expression of ACTA2 and type I collagen $\alpha 1$ chain (COL1A1) while decreasing GPM6A. Conversion of MCs into myofibroblasts was blocked by the inhibition of TGF- $\beta$ receptor or SMAD3, suggesting that a canonical TGF- $\beta /$ SMAD3 pathway is involved in this process. Epithelial cells undergo EMT and acquire a mesenchymal cell phenotype. During this process, TGF- $\beta$ is known to induce Snail transcription repressors and to suppress E-cadherin expression. However, liver MCs do not express E-cadherin, and the expression of Snail is low. ${ }^{10}$ Because MCs are mesodermal in origin and have an intermediate phenotype between epithelial cells and mesenchymal cells, we call the change of MCs into fibroblastic cells mesothelial-mesenchymal transition (MMT). ${ }^{10}$ The cellular mechanisms of MMT might not be identical to what we know about EMT in epithelial cells. Although the details of the downstream pathways of TGF- $\beta$ signaling have not yet been clarified, suppression of TGF- $\beta$ signaling with a soluble form of TGF- $\beta$ type II receptor resulted in decreased MMT in mouse liver fibrosis. ${ }^{10}$ Furthermore, conditional deletion of TGF- $\beta$ type II receptor also reduced their conversion into fibrosis. ${ }^{15,111}$ Thus, TGF- $\beta$ signaling is responsible for the conversion of MCs into myofibroblasts both in vitro and
A

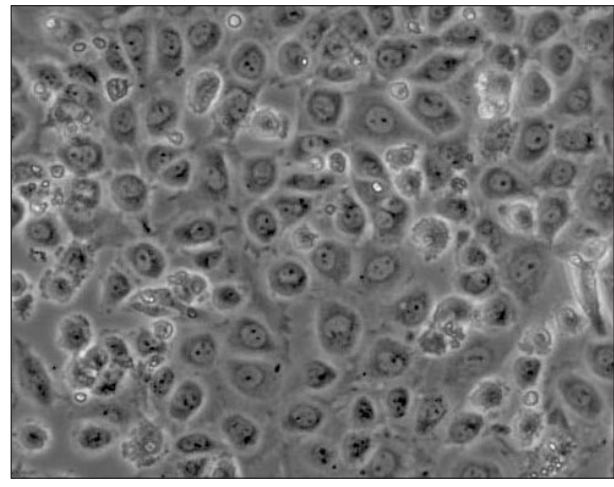

B

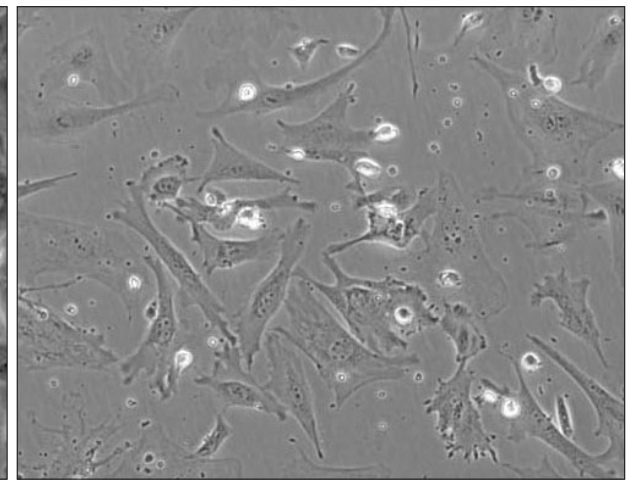

Fig. 6. Primary culture of liver mesothelial cells (MCs). (A) MCs isolated from adult mouse livers form epithelial colonies in culture. (B) Transforming growth factor $\beta$ (TGF- $\beta$ ) treatment induces the mesothelial-mesenchymal transition. 
in vivo.

\section{SECRETORY FUNCTION OF MCS IN PARTIAL HEPATECTOMY AND RESECTION}

The liver has remarkable regenerative potential. ${ }^{112}$ After 70\% partial hepatectomy, hepatocytes start DNA synthesis, and the liver mass returns to the original size by the proliferation and hypertrophy of hepatocytes. ${ }^{113}$ Lineage tracing of WT1+ MCs showed no migration of MCs into the regenerating liver after $70 \%$ partial hepatectomy. ${ }^{10}$ In the regenerating liver, MCs secrete PTN and MDK and support the proliferation of hepatocytes. ${ }^{114}$ PTN- or MDK-knockout mice showed decreased proliferation of hepatocytes in the regenerating liver. ${ }^{115}$ It remains to be clarified how MCs sense regeneration stimuli by partial hepatectomy and how they coordinate proliferation with growing hepatocytes.

MCs secrete a lubricating fluid and have antiadhesive properties. After resection of liver lobes, the resected liver surface often adheres to the other liver lobes or organs, requiring reoperation to remove adhesions between organs. Suzuki et al. ${ }^{116}$ developed an adhesion model of liver surgery. After electrocauterization of rat liver lobes, the cauterized lobe formed adhesions with the intact liver lobes. Interestingly, the necrotic area of the cauterized liver lobe rapidly induced the denudation of MCs from the opposite intact lobe, as well as adhesion with the deposition of fibrin. The finding of molecules inducing denudation of MCs will be important to designing anti-adhesion drugs. An MC sheet created using fetal livers prevented adhesion between the liver and body wall after surgery. ${ }^{114}$ Thus, MCs might be useful for the prevention of organ adhesion and for supporting regeneration of the liver after surgery.

\section{MESOTHELIOMA}

MCs are the source of malignant mesothelioma. Mesothelioma is rare, but exposure to asbestos has increased the risk of mesothelioma in the lung worldwide. Mesothelioma in the liver is extremely rare in humans, but it is experimentally inducible in rats by intraperitoneal injection of asbestos. ${ }^{117,118}$ Ovarian carcinoma cells attached to MCs in the peritoneal cavity and induced fibroblastic conversion of MCs. ${ }^{119}$ These MC-derived cancer-associated fibroblasts might provide a niche for the growth of ovarian carcinoma. Ovarian cancer cells induced a mesenchymal phenotype in MCs via TGF- $\beta{ }^{120}$ Noting is known whether liver MCs also participate in cancer metastasis.

\section{FUTURE DIRECTIONS}

Although MCs were once considered a simple barrier of the organs, recent studies have indicated that MCs dynamically change their phenotype during liver development, injury, and regeneration. Further studies are necessary to understand how
MCs coordinate and regulate liver development and growth with other liver cell types in embryogenesis. During liver fibrosis, MCs contribute to the generation of myofibroblasts near the liver surface. Although TGF- $\beta$ has been shown to induce MMT, it is unclear how MCs sense hepatocyte injury. Elucidation of biological and molecular changes involved in MC activation and fibrogenesis will contribute to the development of novel approaches for prevention and therapy of liver fibrosis. It will also be interesting to examine whether myofibroblasts from different cell sources play different roles in fibrogenesis. ${ }^{121}$ Further studies are necessary to understand how the semi-permeable barrier function of liver MCs is changed in liver fibrosis and how it is involved in ascites formation in cirrhosis.

\section{CONFLICTS OF INTEREST}

No potential conflict of interest relevant to this article are reported.

\section{ACKNOWLEDGEMENTS}

This work was supported by NIH grant R01AA020753 (to K.A.), pilot project funding (to K.A.) from P50AA011999, pilot project funding (to K.A.) from P30DK048522, training program funding (to I.L.) from T32HD060549, and the Robert E. and May R. Wright foundation award (to K.A.).

\section{REFERENCES}

1. Minot CS. The mesoderm and the coelom of vertebrates. Am Nat 1890;24:877-898.

2. Mutsaers SE. Mesothelial cells: their structure, function and role in serosal repair. Respirology 2002;7:171-191.

3. Jones JS. Pathology of the mesothelium. London: SpringerVerlag, 1987.

4. Simionescu M, Simionescu N. Organization of cell junctions in the peritoneal mesothelium. J Cell Biol 1977;74:98-110.

5. Yung S, Thomas GJ, Stylianou E, Williams JD, Coles GA, Davies M. Source of peritoneal proteoglycans: human peritoneal mesothelial cells synthesize and secrete mainly small dermatan sulfate proteoglycans. Am J Pathol 1995;146:520-529.

6. Jonjić N, Peri G, Bernasconi S, et al. Expression of adhesion molecules and chemotactic cytokines in cultured human mesothelial cells. J Exp Med 1992;176:1165-1174.

7. Coulomb-L'Hermin A, Amara A, Schiff C, et al. Stromal cell-derived factor 1 (SDF-1) and antenatal human B cell lymphopoiesis: expression of SDF-1 by mesothelial cells and biliary ductal plate epithelial cells. Proc Natl Acad Sci U S A 1999;96:8585-8590.

8. Krediet RT, Struijk DG. Peritoneal changes in patients on longterm peritoneal dialysis. Nat Rev Nephrol 2013;9:419-429.

9. Chapman GB, Eagles DA. Ultrastructural features of Glisson's capsule and the overlying mesothelium in rat, monkey and pike 
liver. Tissue Cell 2007;39:343-351.

10. Li Y, Wang J, Asahina K. Mesothelial cells give rise to hepatic stellate cells and myofibroblasts via mesothelial-mesenchymal transition in liver injury. Proc Natl Acad Sci U S A 2013;110: 2324-2329.

11. Liang Y, Sasaki K. Expression of adhesion molecules relevant to leukocyte migration on the microvilli of liver peritoneal mesothelial cells. Anat Rec 2000;258:39-46.

12. Mutsaers SE. The mesothelial cell. Int J Biochem Cell Biol 2004; 36:9-16.

13. Shiojiri N, Niwa T, Sugiyama Y, Koike T. Preferential expression of connexin37 and connexin40 in the endothelium of the portal veins during mouse liver development. Cell Tissue Res 2006;324:547-552.

14. Bhunchet E, Wake K. Role of mesenchymal cell populations in porcine serum-induced rat liver fibrosis. Hepatology 1992;16: 1452-1473.

15. Lua I, Li Y, Pappoe LS, Asahina K. Myofibroblastic conversion and regeneration of mesothelial cells in peritoneal and liver fibrosis. Am J Pathol 2015;185:3258-3273.

16. Friedman SL. Hepatic stellate cells: protean, multifunctional, and enigmatic cells of the liver. Physiol Rev 2008;88:125-172.

17. Yin C, Evason KJ, Asahina K, Stainier DY. Hepatic stellate cells in liver development, regeneration, and cancer. J Clin Invest 2013; 123:1902-1910.

18. Thomason RT, Bader DM, Winters NI. Comprehensive timeline of mesodermal development in the quail small intestine. Dev Dyn 2012;241:1678-1694.

19. Winters NI, Thomason RT, Bader DM. Identification of a novel developmental mechanism in the generation of mesothelia. Development 2012;139:2926-2934.

20. Asahina K, Tsai SY, Li P, et al. Mesenchymal origin of hepatic stellate cells, submesothelial cells, and perivascular mesenchymal cells during mouse liver development. Hepatology 2009;49:9981011.

21. Lua I, James D, Wang J, Wang KS, Asahina K. Mesodermal mesenchymal cells give rise to myofibroblasts, but not epithelial cells, in mouse liver injury. Hepatology 2014;60:311-322.

22. Winters NI, Williams AM, Bader DM. Resident progenitors, not exogenous migratory cells, generate the majority of visceral mesothelium in organogenesis. Dev Biol 2014;391:125-132.

23. Douarin NM. An experimental analysis of liver development. Med Biol 1975;53:427-455.

24. Jung J, Zheng M, Goldfarb M, Zaret KS. Initiation of mammalian liver development from endoderm by fibroblast growth factors. Science 1999;284:1998-2003.

25. Rossi JM, Dunn NR, Hogan BL, Zaret KS. Distinct mesodermal signals, including BMPs from the septum transversum mesenchyme, are required in combination for hepatogenesis from the endoderm. Genes Dev 2001;15:1998-2009.

26. Zorn AM. Liver development. Cambridge: StemBook, 2008.

27. Si-Tayeb K, Lemaigre FP, Duncan SA. Organogenesis and devel- opment of the liver. Dev Cell 2010;18:175-189.

28. Enzan H, Himeno H, Hiroi M, Kiyoku H, Saibara T, Onishi S. Development of hepatic sinusoidal structure with special reference to the Ito cells. Microsc Res Tech 1997;39:336-349.

29. Asahina K, Zhou B, Pu WT, Tsukamoto H. Septum transversumderived mesothelium gives rise to hepatic stellate cells and perivascular mesenchymal cells in developing mouse liver. Hepatology 2011;53:983-995.

30. Nahirney PC, Mikawa T, Fischman DA. Evidence for an extracellular matrix bridge guiding proepicardial cell migration to the myocardium of chick embryos. Dev Dyn 2003;227:511-523.

31. Schulte I, Schlueter J, Abu-Issa R, Brand T, Männer J. Morphological and molecular left-right asymmetries in the development of the proepicardium: a comparative analysis on mouse and chick embryos. Dev Dyn 2007;236:684-695.

32. Mikawa T, Gourdie RG. Pericardial mesoderm generates a population of coronary smooth muscle cells migrating into the heart along with ingrowth of the epicardial organ. Dev Biol 1996;174: 221-232.

33. Wessels A, Pérez-Pomares JM. The epicardium and epicardially derived cells (EPDCs) as cardiac stem cells. Anat Rec A Discov Mol Cell Evol Biol 2004;276:43-57.

34. Cai CL, Martin JC, Sun Y, et al. A myocardial lineage derives from Tbx18 epicardial cells. Nature 2008;454:104-108.

35. Zhou B, Ma Q, Rajagopal S, et al. Epicardial progenitors contribute to the cardiomyocyte lineage in the developing heart. Nature 2008;454:109-113.

36. Katz TC, Singh MK, Degenhardt K, et al. Distinct compartments of the proepicardial organ give rise to coronary vascular endothelial cells. Dev Cell 2012;22:639-650.

37. Watt AJ, Battle MA, Li J, Duncan SA. GATA4 is essential for formation of the proepicardium and regulates cardiogenesis. Proc Natl Acad Sci U S A 2004;101:12573-12578.

38. Rojas A, De Val S, Heidt AB, Xu SM, Bristow J, Black BL. Gata4 expression in lateral mesoderm is downstream of BMP4 and is activated directly by Forkhead and GATA transcription factors through a distal enhancer element. Development 2005;132:34053417.

39. Delgado I, Carrasco M, Cano E, et al. GATA4 loss in the septum transversum mesenchyme promotes liver fibrosis in mice. Hepatology 2014;59:2358-2370.

40. Peterson RS, Lim L, Ye H, Zhou H, Overdier DG, Costa RH. The winged helix transcriptional activator HFH-8 is expressed in the mesoderm of the primitive streak stage of mouse embryos and its cellular derivatives. Mech Dev 1997;69:53-69.

41. Kolterud A, Wandzioch E, Carlsson L. Lhx2 is expressed in the septum transversum mesenchyme that becomes an integral part of the liver and the formation of these cells is independent of functional Lhx2. Gene Expr Patterns 2004;4:521-528.

42. Barnes RM, Firulli BA, VanDusen NJ, et al. Hand2 loss-of-function in Hand1-expressing cells reveals distinct roles in epicardial and coronary vessel development. Circ Res 2011;108:940-949. 
43. Lints TJ, Hartley L, Parsons LM, Harvey RP. Mesoderm-specific expression of the divergent homeobox gene Hlx during murine embryogenesis. Dev Dyn 1996;205:457-470.

44. Saito Y, Kojima T, Takahashi N. Mab2112 is essential for embryonic heart and liver development. PLoS One 2012;7:e32991.

45. Christoffels VM, Mommersteeg MT, Trowe MO, et al. Formation of the venous pole of the heart from an Nkx2-5-negative precursor population requires Tbx18. Circ Res 2006;98:1555-1563.

46. Svensson EC, Tufts RL, Polk CE, Leiden JM. Molecular cloning of FOG-2: a modulator of transcription factor GATA-4 in cardiomyocytes. Proc Natl Acad Sci U S A 1999;96:956-961.

47. Berg T, DeLanghe S, Al Alam D, Utley S, Estrada J, Wang KS. beta-Catenin regulates mesenchymal progenitor cell differentiation during hepatogenesis. J Surg Res 2010;164:276-285.

48. Onitsuka I, Tanaka M, Miyajima A. Characterization and functional analyses of hepatic mesothelial cells in mouse liver development. Gastroenterology 2010;138:1525-1535.e6.

49. Ijpenberg A, Pérez-Pomares JM, Guadix JA, et al. Wt1 and retinoic acid signaling are essential for stellate cell development and liver morphogenesis. Dev Biol 2007;312:157-170.

50. Kreidberg JA, Sariola H, Loring JM, et al. WT-1 is required for early kidney development. Cell 1993;74:679-691.

51. Guadix JA, Ruiz-Villalba A, Lettice L, et al. Wt1 controls retinoic acid signalling in embryonic epicardium through transcriptional activation of Raldh2. Development 2011;138:1093-1097.

52. Hentsch B, Lyons I, Li R, et al. Hlx homeo box gene is essential for an inductive tissue interaction that drives expansion of embryonic liver and gut. Genes Dev 1996;10:70-79.

53. Asahina K, Sato H, Yamasaki C, et al. Pleiotrophin/heparinbinding growth-associated molecule as a mitogen of rat hepatocytes and its role in regeneration and development of liver. Am J Pathol 2002;160:2191-2205.

54. Asahina K. Hepatic stellate cell progenitor cells. J Gastroenterol Hepatol 2012;27 Suppl 2:80-84.

55. Rinkevich Y, Mori T, Sahoo D, Xu PX, Bermingham JR Jr, Weissman IL. Identification and prospective isolation of a mesothelial precursor lineage giving rise to smooth muscle cells and fibroblasts for mammalian internal organs, and their vasculature. Nat Cell Biol 2012;14:1251-1260.

56. Loo CK, Wu XJ. Origin of stellate cells from submesothelial cells in a developing human liver. Liver Int 2008;28:1437-1445.

57. Pérez-Pomares JM, Carmona R, González-Iriarte M, Macías D, Guadix JA, Muñoz-Chápuli R. Contribution of mesotheliumderived cells to liver sinusoids in avian embryos. Dev Dyn 2004;229:465-474.

58. Yin C, Evason KJ, Maher JJ, Stainier DY. The basic helix-loophelix transcription factor, heart and neural crest derivatives expressed transcript 2, marks hepatic stellate cells in zebrafish: analysis of stellate cell entry into the developing liver. Hepatology 2012;56:1958-1970.

59. Armulik A, Genové G, Betsholtz C. Pericytes: developmental, physiological, and pathological perspectives, problems, and promises. Dev Cell 2011;21:193-215.

60. Que J, Wilm B, Hasegawa H, Wang F, Bader D, Hogan BL. Mesothelium contributes to vascular smooth muscle and mesenchyme during lung development. Proc Natl Acad Sci U S A 2008;105: 16626-16630.

61. Cano E, Carmona R, Muñoz-Chápuli R. Wt1-expressing progenitors contribute to multiple tissues in the developing lung. Am J Physiol Lung Cell Mol Physiol 2013;305:L322-L332.

62. Dixit R, Ai X, Fine A. Derivation of lung mesenchymal lineages from the fetal mesothelium requires hedgehog signaling for mesothelial cell entry. Development 2013;140:4398-4406.

63. Wilm B, Ipenberg A, Hastie ND, Burch JB, Bader DM. The serosal mesothelium is a major source of smooth muscle cells of the gut vasculature. Development 2005;132:5317-5328.

64. Carmona R, Cano E, Mattiotti A, Gaztambide J, Muñoz-Chápuli R. Cells derived from the coelomic epithelium contribute to multiple gastrointestinal tissues in mouse embryos. PLoS One 2013;8: e55890.

65. Chau YY, Bandiera R, Serrels A, et al. Visceral and subcutaneous fat have different origins and evidence supports a mesothelial source. Nat Cell Biol 2014;16:367-375.

66. Uchio K, Tuchweber B, Manabe N, Gabbiani G, Rosenbaum J, Desmoulière A. Cellular retinol-binding protein-1 expression and modulation during in vivo and in vitro myofibroblastic differentiation of rat hepatic stellate cells and portal fibroblasts. Lab Invest 2002;82:619-628.

67. Li Z, Dranoff JA, Chan EP, Uemura M, Sévigny J, Wells RG. Transforming growth factor-beta and substrate stiffness regulate portal fibroblast activation in culture. Hepatology 2007;46:12461256.

68. Wells RG. The portal fibroblast: not just a poor man's stellate cell. Gastroenterology 2014;147:41-47.

69. Saga Y, Miyagawa-Tomita S, Takagi A, Kitajima S, Miyazaki $\mathrm{Ji}$, Inoue T. MesP1 is expressed in the heart precursor cells and required for the formation of a single heart tube. Development 1999;126:3437-3447.

70. Hernandez-Gea V, Friedman SL. Pathogenesis of liver fibrosis. Annu Rev Pathol 2011;6:425-456.

71. Schuppan D, Kim YO. Evolving therapies for liver fibrosis. J Clin Invest 2013;123:1887-1901.

72. Pellicoro A, Ramachandran P, Iredale JP, Fallowfield JA. Liver fibrosis and repair: immune regulation of wound healing in a solid organ. Nat Rev Immunol 2014;14:181-194.

73. Cassiman D, Barlow A, Vander Borght S, Libbrecht L, Pachnis V. Hepatic stellate cells do not derive from the neural crest. J Hepatol 2006;44:1098-1104.

74. Chagraoui J, Lepage-Noll A, Anjo A, Uzan G, Charbord P. Fetal liver stroma consists of cells in epithelial-to-mesenchymal transition. Blood 2003;101:2973-2982.

75. Conigliaro A, Amicone L, Costa V, et al. Evidence for a common progenitor of epithelial and mesenchymal components of the liver. Cell Death Differ 2013;20:1116-1123. 
76. Knittel T, Kobold D, Saile B, et al. Rat liver myofibroblasts and hepatic stellate cells: different cell populations of the fibroblast lineage with fibrogenic potential. Gastroenterology 1999;117: 1205-1221.

77. Cassiman D, Libbrecht L, Desmet V, Denef C, Roskams T. Hepatic stellate cell/myofibroblast subpopulations in fibrotic human and rat livers. J Hepatol 2002;36:200-209.

78. Lemoinne S, Cadoret A, El Mourabit H, Thabut D, Housset C. Origins and functions of liver myofibroblasts. Biochim Biophys Acta 2013;1832:948-954.

79. Iwaisako K, Jiang C, Zhang M, et al. Origin of myofibroblasts in the fibrotic liver in mice. Proc Natl Acad Sci U S A 2014;111: E3297-E3305.

80. Baba S, Fujii H, Hirose T, et al. Commitment of bone marrow cells to hepatic stellate cells in mouse. J Hepatol 2004;40:255-260.

81. Forbes SJ, Russo FP, Rey V, et al. A significant proportion of myofibroblasts are of bone marrow origin in human liver fibrosis. Gastroenterology 2004;126:955-963.

82. Kisseleva T, Uchinami H, Feirt N, et al. Bone marrow-derived fibrocytes participate in pathogenesis of liver fibrosis. J Hepatol 2006;45:429-438.

83. Higashiyama R, Moro T, Nakao S, et al. Negligible contribution of bone marrow-derived cells to collagen production during hepatic fibrogenesis in mice. Gastroenterology 2009;137:1459-1466.e1.

84. Sicklick JK, Choi SS, Bustamante M, et al. Evidence for epithelialmesenchymal transitions in adult liver cells. Am J Physiol Gastrointest Liver Physiol 2006;291:G575-G583.

85. Kaimori A, Potter J, Kaimori JY, Wang C, Mezey E, Koteish A. Transforming growth factor-beta1 induces an epithelial-to-mesenchymal transition state in mouse hepatocytes in vitro. J Biol Chem 2007;282:22089-22101.

86. Zeisberg M, Yang C, Martino M, et al. Fibroblasts derive from hepatocytes in liver fibrosis via epithelial to mesenchymal transition. J Biol Chem 2007;282:23337-23347.

87. Nitta T, Kim JS, Mohuczy D, Behrns KE. Murine cirrhosis induces hepatocyte epithelial mesenchymal transition and alterations in survival signaling pathways. Hepatology 2008;48:909-919.

88. Rygiel KA, Robertson H, Marshall HL, et al. Epithelial-mesenchymal transition contributes to portal tract fibrogenesis during human chronic liver disease. Lab Invest 2008;88:112-123.

89. Scholten D, Osterreicher CH, Scholten A, et al. Genetic labeling does not detect epithelial-to-mesenchymal transition of cholangiocytes in liver fibrosis in mice. Gastroenterology 2010;139:987998.

90. Taura K, Miura K, Iwaisako K, et al. Hepatocytes do not undergo epithelial-mesenchymal transition in liver fibrosis in mice. Hepatology 2010;51:1027-1036.

91. Chu AS, Diaz R, Hui JJ, et al. Lineage tracing demonstrates no evidence of cholangiocyte epithelial-to-mesenchymal transition in murine models of hepatic fibrosis. Hepatology 2011;53:16851695.

92. Xie G, Diehl AM. Evidence for and against epithelial-to-mesen- chymal transition in the liver. Am J Physiol Gastrointest Liver Physiol 2013;305:G881-G890.

93. Miyajima A, Tanaka M, Itoh T. Stem/progenitor cells in liver development, homeostasis, regeneration, and reprogramming. Cell Stem Cell 2014;14:561-574.

94. Tarlow BD, Pelz C, Naugler WE, et al. Bipotential adult liver progenitors are derived from chronically injured mature hepatocytes. Cell Stem Cell 2014;15:605-618.

95. Yanger K, Knigin D, Zong Y, et al. Adult hepatocytes are generated by self-duplication rather than stem cell differentiation. Cell Stem Cell 2014;15:340-349.

96. Yang L, Jung Y, Omenetti A, et al. Fate-mapping evidence that hepatic stellate cells are epithelial progenitors in adult mouse livers. Stem Cells 2008;26:2104-2113.

97. Michelotti GA, Xie G, Swiderska M, et al. Smoothened is a master regulator of adult liver repair. J Clin Invest 2013;123:2380-2394.

98. Swiderska-Syn M, Syn WK, Xie G, et al. Myofibroblastic cells function as progenitors to regenerate murine livers after partial hepatectomy. Gut 2014;63:1333-1344.

99. Mederacke I, Hsu CC, Troeger JS, et al. Fate tracing reveals hepatic stellate cells as dominant contributors to liver fibrosis independent of its aetiology. Nat Commun 2013;4:2823.

100. Hinz B, Phan SH, Thannickal VJ, et al. Recent developments in myofibroblast biology: paradigms for connective tissue remodeling. Am J Pathol 2012;180:1340-1355.

101. Aroeira LS, Aguilera A, Sánchez-Tomero JA, et al. Epithelial to mesenchymal transition and peritoneal membrane failure in peritoneal dialysis patients: pathologic significance and potential therapeutic interventions. J Am Soc Nephrol 2007;18:2004-2013.

102. Devuyst 0, Margetts PJ, Topley N. The pathophysiology of the peritoneal membrane. J Am Soc Nephrol 2010;21:1077-1085.

103. Korte MR, Sampimon DE, Betjes MG, Krediet RT. Encapsulating peritoneal sclerosis: the state of affairs. Nat Rev Nephrol 2011; 7:528-538.

104. Yáñez-Mó M, Lara-Pezzi E, Selgas R, et al. Peritoneal dialysis and epithelial-to-mesenchymal transition of mesothelial cells. N Engl J Med 2003;348:403-413.

105. Chen YT, Chang YT, Pan SY, et al. Lineage tracing reveals distinctive fates for mesothelial cells and submesothelial fibroblasts during peritoneal injury. J Am Soc Nephrol 2014;25:2847-2858.

106. Karki S, Surolia R, Hock TD, et al. Wilms' tumor 1 (Wt1) regulates pleural mesothelial cell plasticity and transition into myofibroblasts in idiopathic pulmonary fibrosis. FASEB J 2014;28:11221131.

107. Shelton EL, Galindo CL, Williams CH, Pfaltzgraff E, Hong CC, Bader DM. Autotaxin signaling governs phenotypic heterogeneity in visceral and parietal mesothelia. PLoS One 2013;8:e69712.

108. Kim KH, Stellmach V, Javors J, Fuchs E. Regulation of human mesothelial cell differentiation: opposing roles of retinoids and epidermal growth factor in the expression of intermediate filament proteins. J Cell Biol 1987;105(6 Pt 2):3039-3051.

109. Faris RA, McBride A, Yang L, Affigne S, Walker C, Cha CJ. Isola- 
tion, propagation, and characterization of rat liver serosal mesothelial cells. Am J Pathol 1994;145:1432-1443.

110. Lamouille S, Xu J, Derynck R. Molecular mechanisms of epithelial-mesenchymal transition. Nat Rev Mol Cell Biol 2014;15:178196.

111. Li Y, Lua I, French SW, Asahina K. Role of TGF-beta signaling in differentiation of mesothelial cells to vitamin A-poor hepatic stellate cells in liver fibrosis. Am J Physiol Gastrointest Liver Physiol 2016;310:G262-G272.

112. Michalopoulos GK. Liver regeneration: alternative epithelial pathways. Int J Biochem Cell Biol 2011;43:173-179.

113. Miyaoka Y, Ebato K, Kato H, Arakawa S, Shimizu S, Miyajima A. Hypertrophy and unconventional cell division of hepatocytes underlie liver regeneration. Curr Biol 2012;22:1166-1175.

114. Inagaki NF, Inagaki FF, Kokudo N, Miyajima A. Use of mouse liver mesothelial cells to prevent postoperative adhesion and promote liver regeneration after hepatectomy. J Hepatol 2015;62: 1141-1147.

115. Ochiai K, Muramatsu H, Yamamoto S, Ando H, Muramatsu T. The role of midkine and pleiotrophin in liver regeneration. Liver Int 2004;24:484-491.
116. Suzuki T, Kono T, Bochimoto H, Hira Y, Watanabe T, Furukawa H. An injured tissue affects the opposite intact peritoneum during postoperative adhesion formation. Sci Rep 2015;5:7668.

117. Serter A, Buyukpinarbasili N, Karatepe 0, Kocakoc E. An unusual liver mass: primary malignant mesothelioma of the liver: CT and MRI findings and literature review. Jpn J Radiol 2015;33:102106.

118. Jiang L, Akatsuka S, Nagai H, et al. Iron overload signature in chrysotile-induced malignant mesothelioma. J Pathol 2012;228: 366-377.

119. Sandoval P, Jiménez-Heffernan JA, Rynne-Vidal Á, et al. Carcinoma-associated fibroblasts derive from mesothelial cells via mesothelial-to-mesenchymal transition in peritoneal metastasis. J Pathol 2013;231:517-531.

120. Kenny HA, Chiang CY, White EA, et al. Mesothelial cells promote early ovarian cancer metastasis through fibronectin secretion. J Clin Invest 2014;124:4614-4628.

121. Lua I, Li Y, Zagory JA, et al. Characterization of hepatic stellate cells, portal fibroblasts, and mesothelial cells in normal and fibrotic livers. J Hepatol 2016, in press. 